\title{
Prediction of Treatment Resistance in Conservative Treatment of Osteoporotic Vertebral Fractures Using Lateral Plain Dynamic Loading Radiographs
}

Toru Funayama $^{1^{*}}$, Toshinori Tsukanishi ${ }^{2}$, Tetsuya Abe ${ }^{1}$, Hiroshi Kumagai ${ }^{1}$, Shigeo Izawa ${ }^{2}$, Hiroshi Noguchi ${ }^{1}$, Kengo Fujii ${ }^{1}$, Yousuke Shibao ${ }^{1}$, Masao Koda ${ }^{1}$ and Masashi Yamazaki ${ }^{1}$

${ }^{1}$ Department of Orthopedic Surgery, University of Tsukuba, Tsukuba, Japan

${ }^{2}$ Department of Orthopedic Surgery, Kenpoku Medical Center, Takahagi Kyodo Hospital, Japan

*Corresponding author: Toru Funayama, Department of Orthopedic Surgery, University of Tsukuba, Tsukuba, Japan, Tel: +81-29-853-3219; Fax: +81-29-853-3162; Email: funatoru3@md.tsukuba.ac.jp

Rec Date: December 10, 2017; Acc Date: December 26, 2017; Pub Date: December 31, 2017

Copyright: @ 2017 Funayama T, et al. This is an open-access article distributed under the terms of the creative commons attribution license, which permits unrestricted use, distribution, and reproduction in any medium, provided the original author and source are credited.

\begin{abstract}
Purpose: To elucidate the association between computed tomography (CT)/magnetic resonance imaging (MRI) and radiographic findings of fractured vertebral body instability in patients with osteoporotic vertebral fractures and to clarify whether resistance to conservative treatment can be evaluated on the basis of dynamic loading radiography.

Methods: Seventy-eight patients aged $\geq 65$ years who underwent conservative treatment for osteoporotic single vertebral fractures of the thoracolumbar junction were divided into the conservative treatment-resistant group (18 patients) and control group (60 patients). We evaluated the accuracy of the prediction of resistance to conservative treatment on the basis of the CT/MRI findings and the difference in compression rates between standing and supine positions at the time of the first visit. The differences in compression rates (\%) were compared between the two groups. In addition, a receiver operating characteristics (ROC) curve was drawn to evaluate the accuracy of the prediction of resistance to conservative treatment.
\end{abstract}

Results: In patients without (47 cases) and with CT findings (31 cases), the mean differences in compression rates $(\%)$ was $8.9 \%$ and $19.1 \%$, respectively $(p=0.0029)$. The mean differences in compression rates $(\%)$ of patients without (60 cases) and with MRI findings (18 cases) was $9.7 \%$ and $24.0 \%$, respectively $(p=0.0043)$. The mean differences in compression rates (\%) in the conservative treatment-resistant group was $26.3 \%$, while that in the control group was $9.0(p=0.0066)$. In addition, according to the ROC curve of the difference in compression rate was 0.93 (95\% confidence interval: $0.87-1)$, and when a $20 \%$ difference in compression rate was considered as the threshold value.

Conclusion: Dynamic loading radiography is useful for the evaluation of resistance to conservative treatment in patients with osteoporotic vertebral fractures, and that a compression rate difference of $\geq 20 \%$ predicts resistance to conservative treatment.

Keywords: Osteoporotic vertebral fracture; Thoracolumbar junction; Conservative therapy; Prediction of treatment resistance; Lateral plain dynamic loading radiograph

\section{Introduction}

Osteoporotic Vertebral Fractures (OVFs) are fragility fractures associated with osteoporosis. They have a prevalence of $8 \%$ to $13 \%$ among people in their 60 s and $30 \%$ to $40 \%$ among people in their 70 s in developed countries [1-4]. The probability for a 50-year-old woman to develop a fracture during her lifetime is approximately $40 \%$ [1-4]. With the aging population, the prevalence rate of primary osteoporosis has increased rapidly, and OVFs are becoming more commonplace in orthopedic outpatient departments. Conventionally, OVFs should be managed using conservative treatment and, because various types of evidence-based conservative treatment have been developed and tested, no standard treatment or treatment guidelines have been established. According to previous reports, the majority of clinical fractures with pain develop at the thoracolumbar junction (T11-L2)
$[5,6]$. While bone union can be achieved with conservative treatment in the majority of cases, delayed union and pseudarthrosis occur in approximately $10 \%$ to $35 \%$ of patients [6-8]. In addition, patients who require surgery because of delayed union and pseudarthrosis often have fractures of the thoracolumbar junction [6,9-11]. If complete bone union is not achieved and the patient develops delayed union and pseudarthrosis, most will become bedridden due to persistent with severe pain. In some cases, patients develop delayed neurological deficits in the lower limb due to progressive collapse of the vertebral body. Therefore, in fractures of the thoracolumbar junction, which account for the majority of OVFs, it is important that the factors responsible for resistance to conservative treatment are understood.

The following have been suggested as factors contributing to resistance to conservative treatment: computed tomography (CT) images showing injury to the posterior wall of the vertebral body [12], magnetic resonance imaging (MRI) T2-weighted images showing localized high-signal intensity changes in the vertebral body, or the presence of diffuse low-signal intensity changes on T2-weighted 
images [6]. Although these findings are important, they are qualitative in nature and do not allow for the evaluation of the severity or size of the posterior wall injuries found on CT images or signal intensity changes on MRI images. These imaging tests are therefore limited. Meanwhile, lateral plain dynamic loading radiographs obtained in the standing or sitting position (loaded posture) and supine position (nonloaded posture) allow for the evaluation of vertebral body instability [13-16]. Plain dynamic loading radiography involves taking images of the lateral surface of the vertebral body in both postures and calculating changes in vertebral height between the two. This method is believed to significantly improve the accuracy of the diagnosis of fresh fractures at the time of the first visit [5] compared to conventional bidirectional plain radiography, which is performed in the supine position for anterior-posterior imaging and in the side-lying non-loaded position for lateral imaging [17]. In addition, by using images obtained from plain dynamic loading radiography to measure differences in vertebral collapse rate in the standing/sitting and supine position, instability of the fractured vertebral body in a loaded posture and non-loaded posture can be evaluated.

On the basis of some previous reports $[18,19]$ and our previous clinical experience of large numbers of conservative treatmentresistant cases in patients with considerable vertebral body instability, we established a hypothesis that the quantitative evaluation of vertebral body instability by plain dynamic loading radiographs obtained at the time of the initial visit may allow the prediction of resistance to conservative treatment in OVFs. No study has tested this hypothesis to date. In addition, no previous study has reported any association between quantitative evaluations of vertebral body instability based on dynamic imaging and qualitative findings obtained using CT and MRI. With the above in mind, the purposes of this study were to examine the association between CT/MRI and plain dynamic loading radiography findings at the initial visit as an imaging test for estimating the resistance to conservative treatment in OVFs, and to calculate the threshold of vertebral body instability for predicting resistance to conservative treatment.

\section{Materials and Methods}

This retrospective cohort study was performed with the approval of appropriate ethics committee on clinical research. Seventy-eight consecutive patients aged $\geq 65$ years with a new osteoporosis-related fracture of a single vertebral body at the thoracolumbar junction (T11L2) between April 2012 and March 2015 were enrolled. All patients were admitted to our institute, and started receiving treatment within 2 weeks after injury. We excluded patients with a previous history or complications of conditions that could clearly be diagnosed as secondary osteoporosis, such as those associated with long-term steroid treatment, long-term hemodialysis, and diabetes with poor control (HbA1c 7.5\% or higher at the time of treatment initiation).

At the first visit, all patients were subjected to plain dynamic loading radiography in the standing and supine positions, which provided a definitive diagnosis of a fresh OVF. When standing was impossible because of pain, imaging was performed in the sitting position. Conservative treatment was carried out using a standardized protocol in which all patients were hospitalized and completely rested in bed without any weight bearing to the spine for 2 weeks [20]. The patients were then allowed to leave their hospital bed while wearing a Jewetttype hard corset. In addition, at the time of hospital admission, patients were interviewed regarding their pain (visual analog scale (VAS) score). Bone mineral density testing in lumbar spine was performed, and the measurements were compared with the young adult mean (YAM) value. Finally, CT and MRI were performed early after admission. Teriparatide injection was introduced for all patients as the treatment of osteoporosis.

On the basis of the heights of the anterior wall (A) and posterior wall $(\mathrm{P})$ of the fractured vertebral body on plain dynamic radiographs, the compression rate was calculated as $(1-\mathrm{A} / \mathrm{P}) \times 100(\%)$, and vertebral body instability was defined as the difference in compression rates between the two postures (\%) (Figure 1).

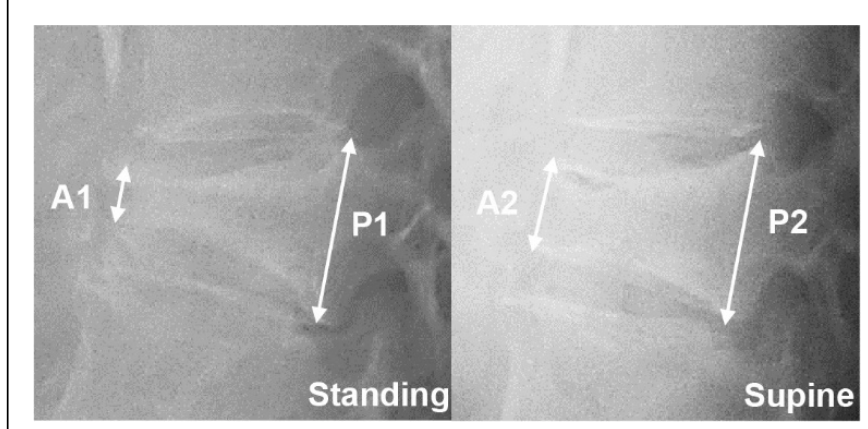

Figure 1: Definition of vertebral body instability. Based on the anterior $(\mathrm{A})$ and posterior $(\mathrm{P})$ wall height, compression rate $=(1-$ $\mathrm{A} / \mathrm{P}) \times 100(\%)$, and vertebral body instability $=(1-\mathrm{A} 1 / \mathrm{P} 1) \times 100$ (\%) $-(1-\mathrm{A} 2 / \mathrm{P} 2) \times 100(\%)$.

\section{Study 1: Relationship between plain dynamic loading radiographs and CT/MRI findings}

To clarify the association between plain dynamic loading radiography findings and CT/MRI findings, the differences in compression rates found on plain dynamic loading radiographs were compared on the basis of evidence of resistance to conservative treatment in CT/MRI findings early after the initiation of treatment. The presence of CT findings of injury to the posterior wall of the vertebral body and MRI findings showing localized high-signal intensity changes in the vertebral body or diffuse low-signal intensity changes on the T2-weighted image were considered to indicate resistance to conservative treatment.

\section{Study 2: Threshold values of dynamic loading radiographs for resistance to conservative treatment}

The conservative treatment-resistant group (group R) included patients whose findings at 3 months after treatment initiation showed delayed union and pain with a severity that had not decreased to less than half of its intensity at the beginning of treatment and patients who were treated surgically because of a delayed improvement in pain. Other patients for whom conservative treatment was successful were defined as the control group (group C). The differences in compression rates (\%) on plain dynamic loading radiographs obtained at the time of the first visit were compared between the two groups. In addition, a receiver operating characteristics (ROC) curve was drawn to evaluate the accuracy of the prediction of resistance to conservative treatment on the basis of the difference in compression rate between the two postures. The maximum value of the sum of sensitivity and specificity was calculated as the threshold value for resistance to conservative treatment. 
Citation: Funayama T, Tsukanishi T, Abe T, Kumagai H, Izawa S, et al. (2017) Prediction of Treatment Resistance in Conservative Treatment of Osteoporotic Vertebral Fractures Using Lateral Plain Dynamic Loading Radiographs. J Spine 6: 404. doi:10.4172/2165-7939.1000404

Page 3 of 6

For statistical analysis, the age, body mass index (BMI), YAM values and VAS were compared between the two groups using t-tests. All other items were compared using Welch's t-test. A p-value $<0.05$ was considered statistically significant. All statistical analyses were performed using the software 'EZR' [21] (Saitama Medical Center, Jichi Medical University, Saitama, Japan).

\section{Results}

We enrolled 78 patients (18 male and 60 female) with a mean age of $80.0 \pm 7.9$ years.

\section{Study 1}

For patients without (47 cases) and with CT findings (31 cases), mean vertebral body instability was $8.9 \pm 6.5 \%$ and $19.1 \pm 13.9 \%$, respectively $(\mathrm{p}=0.0029)$ (Figure 2$)$.

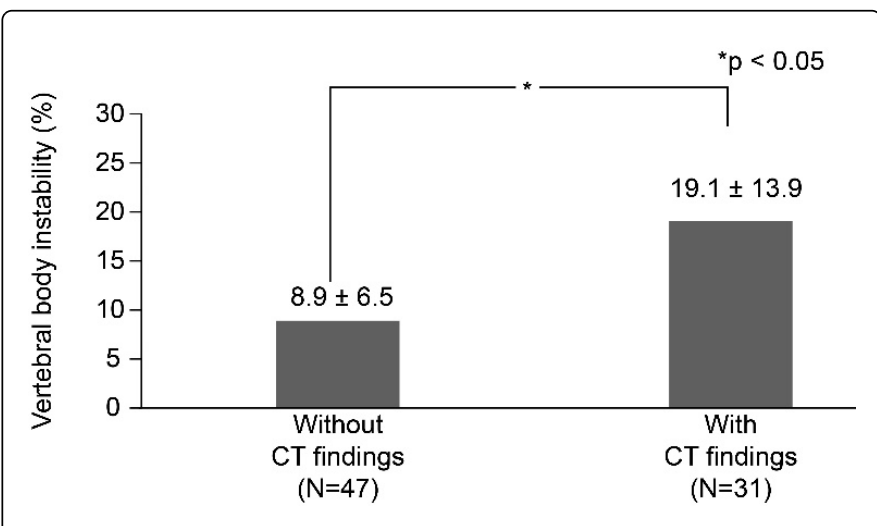

Figure 2: Association between CT findings and vertebral body instability. In patients without CT findings (47 cases) and those with CT findings (31 cases), the mean vertebral body instability was $8.9 \pm 6.5 \%$ and $19.1 \pm 13.9 \%$, respectively $(\mathrm{p}=0.0029)$.
In patients without (60 cases) and with MRI findings (18 cases), mean vertebral body instability was $9.7 \pm 6.8 \%$ and $24.0 \pm 15.4 \%$, respectively $(\mathrm{p}=0.0043)$ (Figure 3 ).

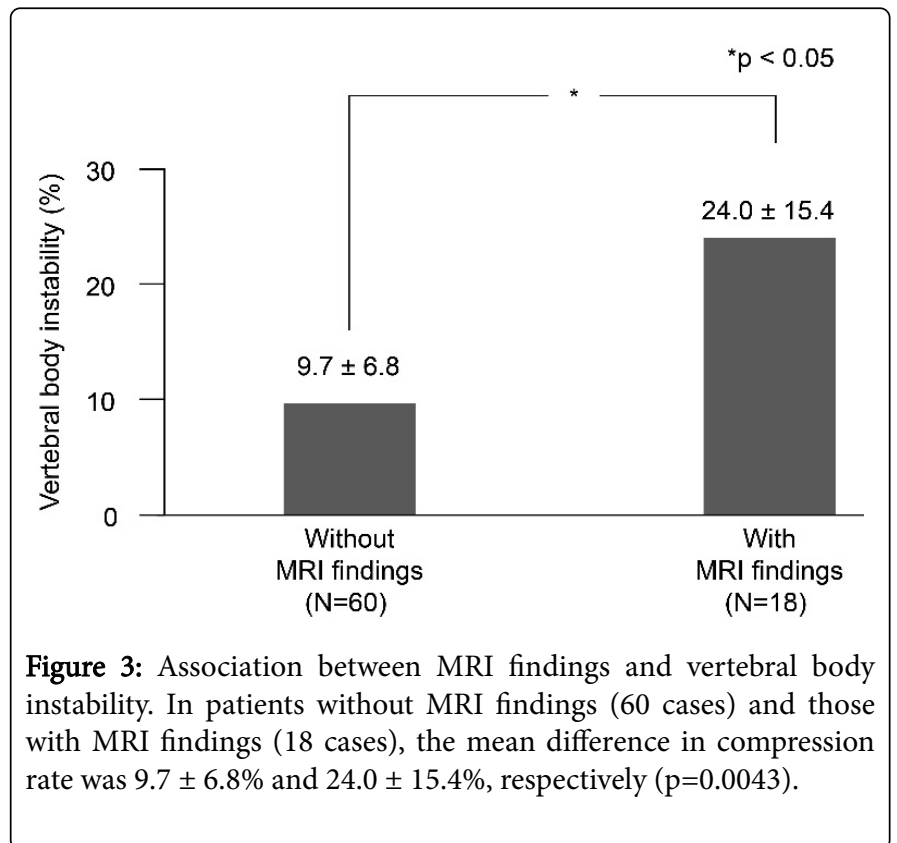

\section{Study 2}

In group C (60 patients) and group $\mathrm{R}$ (18 patients), the male-tofemale ratios were 14:46 and 4:14, respectively. Spinal injuries located at T11, T12, L1, and L2 accounted for four, 18, 30, and eight patients in group $\mathrm{C}$, and two, seven, seven, and two patients in group $\mathrm{R}$. The mean age was $81.0 \pm 8.3$ years in group $C$ and $78.0 \pm 6.1$ years in group $R$ (no significant difference). Furthermore, BMI, YAM value, and VAS score at the time of treatment initiation showed no significant differences (Table 1).

\begin{tabular}{|c|c|c|c|}
\hline Variables & Group C (n=60) & Group R (n=18) & p-value \\
\hline Male: female & $14: 46$ & $4: 14$ & - \\
\hline Spinal level & - & - & - \\
\hline T11 & 4 & 7 & - \\
\hline T12 & 18 & 7 & - \\
\hline L1 & 30 & 2 & - \\
\hline L2 & 8 & $78.0 \pm 6.1$ & 0.19 (N.S.) \\
\hline Age & $81.0 \pm 8.3$ & $22.8 \pm 3.2$ & 0.79 (N.S.) \\
\hline BMI & $22.4 \pm 5.0$ & $74.5 \pm 4.2$ & 0.83 (N.S.) \\
\hline YAM (\%) & $75.0 \pm 7.4$ & $75.0 \pm 19.6$ & 0.45 (N.S.) \\
\hline VAS & $81.0 \pm 20.1$ & & \\
\hline $\begin{array}{l}\text { Group C: Control Group; Group R: Conservative Treatment-Resistant Group; BMl: Body Mass Index; YAM: Young Adult Mean of Bone Mineral Density; VAS: Visual } \\
\text { Analog Scale Score of Pain at the Time of Hospital Admission; N.S.: Not Significant }\end{array}$ \\
\hline
\end{tabular}

Table 1: Summary of patient demographic information. 
Page 4 of 6

Among the 18 patients in group R, 10 were treated surgically. Of those, five were treated by balloon kyphoplasty and the other five by vertebroplasty with posterior pedicle screw instrumentation.

Plain dynamic loading radiographs for the evaluation of resistance to conservative treatment showed that mean vertebral body instability was $9.0 \pm 6.3 \%$ in group $\mathrm{C}$ and $26.3 \pm 14.1 \%$ in group $\mathrm{R}(\mathrm{p}=0.0066)$ (Figure 4).

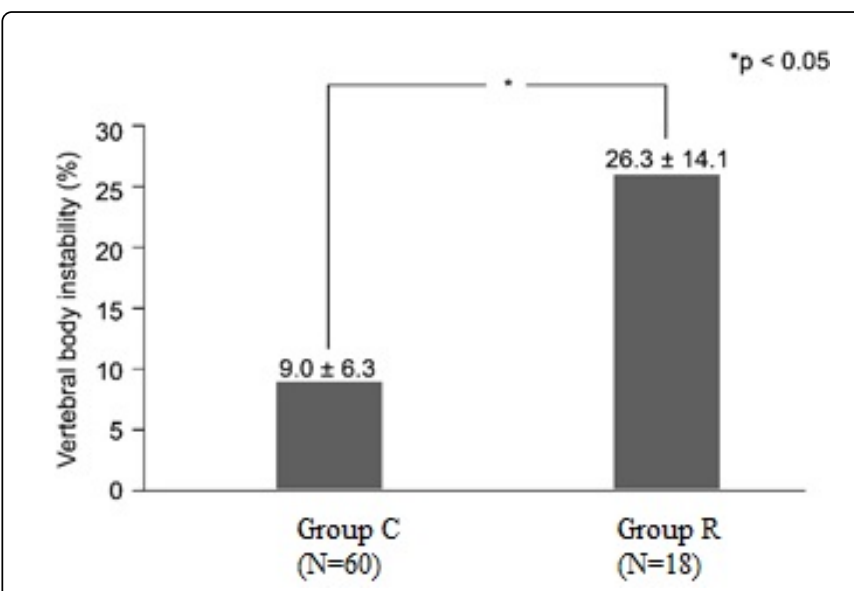

Figure 4: Associations between resistance to conservative treatment and vertebral body instability. The mean vertebral body instability was $9.0 \pm 6.3 \%$ in the Control (Group C) and $26.3 \pm 14.1 \%$ in the Conservative treatment-resistant (Group R) ( $\mathrm{p}=0.0066)$.

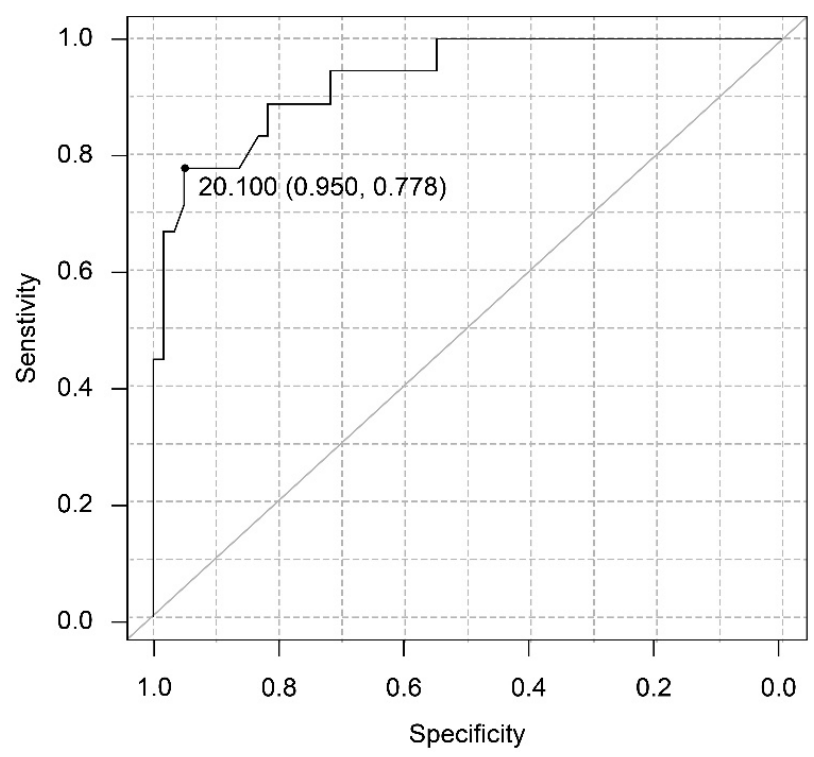

Figure 5: ROC curve analysis of vertebral body instability. At a threshold of $20 \%$ difference in compression rate between the postures, sensitivity was $78 \%$ and specificity was $95 \%$ and the sum of the two was maximized.

In addition, the area under the ROC curve of the difference in compression rate was 0.93 (95\% confidence interval: $0.87-1$ ), and when a $20 \%$ difference in compression rate between the two postures was considered as the threshold value, the sensitivity was $78 \%$ and specificity was $95 \%$; the sum of the two was maximized at this threshold (Figure 5).

\section{Discussion}

OVFs are generally managed using conservative treatment, but because some cases are resistant to this approach, it is crucial that high-risk patients be identified in the early period after treatment initiation. In previous reports, the occurrence of an intravertebral cleft at 6 months after treatment initiation was considered as non-union (pseudarthrosis) $[6,12]$, and in most instances, such cases have been viewed as resistant to conservative treatment. However, in actual clinical settings, pain that persists for approximately 3 months after treatment initiation leads to deterioration of the patient's activities of daily living (ADL) and leaves the patient almost completely bedridden. In addition, in patients whose pain persists, and ADL do not improve despite conservative treatment, surgical treatment at an appropriate timing before the development of pseudarthrosis can lead to a drastic improvement in ADL in some cases. Thus, in the present study, the determination of conservative treatment-resistant cases was based on estimations made at 3 months after treatment initiation.

The results of the present study revealed that vertebral body instability detected on plain dynamic loading radiographs was significantly greater in patients with CT or MRI findings. This is in line with previous reports $[6,12]$. In addition, the present study revealed that there were differences between the groups $\mathrm{R}$ and $\mathrm{C}$ in terms of vertebral body instability detected on plain dynamic loading radiographs taken at the time of the initial visit. Furthermore, the results of this study demonstrated that the quantitative evaluation of vertebral body instability was highly accurate, and allowed for the prediction of resistance to conservative treatment. If the differences in compression rates between the two postures at the time of the first visit are $\geq 20 \%$ and if pain is persistent, conservative treatment will probably failed. Instead, it is preferable to perform surgical treatment at an appropriate timing, even within the first 3 months of treatment.

Plain dynamic loading radiography improves the diagnostic accuracy of OVFs at the time of the initial visit [5]. In addition, this study demonstrated that plain dynamic loading radiography is an extremely useful imaging tool for the quantitative evaluation of vertebral body instability. Plain dynamic loading radiography is relatively simple and easy to perform if there is standard radiographic $\mathrm{X}$-ray equipment available. OFVs are becoming more commonplace in outpatient clinics or departments [22]. In facilities such as outpatient clinics in charge of primary care, which account for the majority of medical institutions and are not equipped with advanced diagnostic imaging devices such as CT and MRI, plain dynamic loading radiography can be utilized as a new way to help with the evaluation of resistance to conservative treatment in fresh OVFs. It is important to note that if the threshold of $20 \%$ is not reached, clinicians can still decide to continue conservative treatment. Although there is no objection to the fact that OVFs should be managed by conservative treatment, some patients are resistant to conservative treatment, especially those with fractures affecting the thoracolumbar junction. Although CT images showing injury to the posterior wall of the vertebral body and MRI T2-weighted images showing localized highsignal-intensity in the vertebral body or diffuse low-signal intensity changes are important for detecting qualitative findings of resistance to conservative treatment $[6,12,23]$, the results of this study indicate that radiographs can be used if the clinical setting is appropriate. In the 
future, treating OVFs in a personalized manner will be made possible by varying the length and characteristics of the bed-rest period in accordance with quantitative findings of vertebral body instability. In other words, conservative treatment will be considered as the first choice for the management of patients with extremely mild vertebral body instability, and in such cases, it may be possible to shorten the period of bed-rest. Furthermore, in cases involving extremely unstable vertebral bodies, conservative treatment should be changed to surgical treatment at an appropriate interval, ideally before pain becomes persistent and ADL deteriorate. Additionally, in evaluating such cases, it is important to possess adequate understanding of the factors responsible for resistance to conservative treatment, for which plain dynamic loading radiographs obtained in the standing and supine positions at treatment initiation can be useful.

This study had several limitations. First, the number of conservative treatment-resistant group was relatively small. Second, the cases included were limited to fractures affecting the thoracolumbar junction, and patients with secondary osteoporosis were excluded; therefore, the findings of the present study cannot be generalized to all kinds of OVFs. However, in clinical settings, particularly in postmenopausal women, secondary osteoporosis accounts for approximately $20 \%$ or $<30 \%$ of all cases of osteoporosis [24,25], and fractures of the thoracolumbar junction account for the majority of OVFs $[5,6]$. Although a larger study is needed to confirm and generalize the results of the present study for all OVF cases, a plain dynamic loading radiography is an extremely useful imaging tool, which allows for the resistance of OVFs to conservative treatment to be quantitatively evaluated based on vertebral body instability in most cases.

\section{Conclusion}

In conclusion, plain dynamic loading radiographs taken at the first visit in the standing and supine positions are useful for the evaluation of resistance to conservative treatment of OVSs in the thoracolumbar junction. The size and magnitude of vertebral body instability detected by dynamic loading radiographs are greatly associated with qualitative CT and MRI findings of resistance to conservative treatment. When the difference in vertebral collapse rates according to body postures during dynamic loading imaging tests is $\geq 20 \%$, resistance to conservative treatment is highly likely and physicians should consider changing the treatment approach to a surgical method if pain is persistent.

\section{Acknowledgements}

We are grateful to Ms. Kanako Suzuki, a doctor's assistant, for her assistance with data collection. This study was partially supported by a grant from the JA Kyosairen Research Fund.

\section{References}

1. Kitazawa A, Kushida K, Yamazaki K, Inoue T (2001) Prevalence of vertebral fractures in a population-based sample in Japan. J Bone Miner Metab 19: 115-118.

2. Ross PD (1997) Clinical consequences of vertebral fractures. Am J Med 103: 30-42.

3. Ross PD, Fujiwara S, Huang C, Davis JW, Epstein RS, et al. (1995) Vertebral fracture prevalence in women in Hiroshima compared to Caucasians or Japanese in the US. Int J Epidemiol 24: 1171-1177.
4. Yoshimura N, Kinoshita H, Danjoh S, Yamada H, Tamaki T, et al. (1995) Prevalence of vertebral fractures in a rural Japanese population. J Epidemiol 5: 171-175

5. Niimi R, Kono T, Nishihara A, Hasegawa M, Matsumine A, et al. (2014) Efficacy of the dynamic radiographs for diagnosing acute osteoporotic vertebral fractures. Osteoporos Int 25: 605-612.

6. Tsujio T, Nakamura H, Terai H, Hoshino M, Namikawa T, et al. (2011) Characteristic radiographic or magnetic resonance images of fresh osteoporotic vertebral fractures predicting potential risk for nonunion: A prospective multicenter study. Spine 36: 1229-1235.

7. Kim DY, Lee SH, Jang JS, Chung SK, Lee HY (2004) Intravertebral vacuum phenomenon in osteoporotic compression fracture: Report of 67 cases with quantitative evaluation of intravertebral instability. J Neurosurg 100: 24-31.

8. Wu CT, Lee SC, Lee ST, Chen JF (2006) Classification of symptomatic osteoporotic compression fractures of the thoracic and lumbar spine. J Clin Neurosci 13: 31-38.

9. Ataka H, Tanno T, Yamazaki M (2009) Posterior instrumented fusion without neural decompression for incomplete neurological deficits following vertebral collapse in the osteoporotic thoracolumbar spine. Eur Spine J 18: 69-76.

10. Katsumi K, Hirano T, Watanabe K, Ohashi M, Yamazaki A, et al. (2016) Surgical treatment for osteoporotic thoracolumbar vertebral collapse using vertebroplasty with posterior spinal fusion: a prospective multicenter study. Int Orthop 40: 2309-2315.

11. Sudo H, Ito M, Abumi K, Kotani Y, Takahata M, et al. (2010) One-stage posterior instrumentation surgery for the treatment of osteoporotic vertebral collapse with neurological deficits. Eur Spine J 19: 907-915.

12. Hoshino M, Tsujio T, Terai H, Namikawa T, Kato M, et al. (2013) Impact of initial conservative treatment interventions on the outcomes of patients with osteoporotic vertebral fractures. Spine 38: 641-648.

13. Kawaguchi S, Horigome K, Yajima H, Oda T, Kii Y (2011) Comparative supine-sitting lateral radiographs identifying incident osteoporotic vertebral fractures. Euro Orthopaedics Traumatol 1: 157-162.

14. McKiernan F, Jensen R, Faciszewski $\mathrm{T}$ (2003) The dynamic mobility of vertebral compression fractures. J Bone Miner Res 18: 24-29.

15. Mehta JS, Reed MR, McVie JL, Sanderson PL (2004) Weight-bearing radiographs in thoracolumbar fractures: Do they influence management? Spine 29: 564-567.

16. Wu MH, Huang TJ, Cheng CC, Li YY, Hsu RW (2010) Role of the supine lateral radiograph of the spine in vertebroplasty for osteoporotic vertebral compression fracture: A prospective study. BMC Musculoskelet Disord 11: 164 .

17. Ito Z, Harada A, Matsui Y, Takemura M, Wakao N, et al. (2006) Can you diagnose for vertebral fracture correctly by plain X-ray?. Osteoporos Int 17: 1584-1591.

18. Suseki K, Takahashi Y, Toyone T, Kito M, Yamashita M, et al. (2013) Risk factor analysis for persistent back pain of intravertebral vacuum cleft after osteoporotic vertebral fracture: Can we predict persistent back pain based on plain radiography? Current Orthopaedic Practice 24: 414-419.

19. Toyone T, Tanaka T, Wada Y, Kamikawa K, Ito M, et al. (2006) Changes in vertebral wedging rate between supine and standing position and its association with back pain: a prospective study in patients with osteoporotic vertebral compression fractures. Spine 31: 2963-2966.

20. Kishikawa $Y$ (2012) Initial non-weight-bearing therapy is important for preventing vertebral body collapse in elderly patients with clinical vertebral fractures. Int J Gen Med 5: 373-380.

21. Kanda Y (2013) Investigation of the freely available easy-to-use software 'EZR' for medical statistics. Bone Marrow Transplant 48: 452-458.

22. Bottai V, Giannotti S, Raffaeta G, Mazzantini M, Casella F, et al. (2016) Under diagnosis of osteoporotic vertebral fractures in patients with fragility fractures: Retrospective analysis of over 300 patients. Clin Cases Miner Bone Metab 13: 119-122. 
Citation: Funayama T, Tsukanishi T, Abe T, Kumagai H, Izawa S, et al. (2017) Prediction of Treatment Resistance in Conservative Treatment of Osteoporotic Vertebral Fractures Using Lateral Plain Dynamic Loading Radiographs. J Spine 6: 404. doi:10.4172/2165-7939.1000404

Page 6 of 6

23. Hashidate H, Kamimura M, Nakagawa H, Takahara K, Uchiyama S (2006) Pseudoarthrosis of vertebral fracture: Radiographic and characteristic clinical features and natural history. J Orthop Sci 11: 28-33.

24. Fitzpatrick LA (2002) Secondary causes of osteoporosis. Mayo Clin Proc 77: 453-468.
25. Walker-Bone K (2012) Recognizing and treating secondary osteoporosis. Nat Rev Rheumatol 8: 480-492. 